\title{
Transatlantica
}

Revue d'études américaines. American Studies Journal

\section{Muddy Waters and the Rolling Stones, Live at the Checkerboard Lounge, Chicago 1981.}

CD/DVD et LP. Eagle Vision, enregistré le 22 novembre $1981 \mathrm{au}$

Checkerboard Lounge de Chicago, sortie le 10 juillet 2012.

Elodie A. Grossi

\section{OpenEdition}

Journals

Édition électronique

URL : https://journals.openedition.org/transatlantica/6110

DOI : $10.4000 /$ transatlantica. 6110

ISSN : $1765-2766$

Éditeur

Association française d'Etudes Américaines (AFEA)

Référence électronique

Elodie A. Grossi, « Muddy Waters and the Rolling Stones, Live at the Checkerboard Lounge, Chicago 1981. », Transatlantica [En ligne], 2 | 2012, mis en ligne le 02 mai 2013, consulté le 06 avril 2023. URL http://journals.openedition.org/transatlantica/6110; DOI : https://doi.org/10.4000/transatlantica. 6110

Ce document a été généré automatiquement le 6 avril 2023.

\section{(i) $(9)$}

Creative Commons - Attribution - Pas d'Utilisation Commerciale - Pas de Modification 4.0 International - CC BY-NC-ND 4.0

https://creativecommons.org/licenses/by-nc-nd/4.0/ 


\section{Muddy Waters and the Rolling Stones, Live at the Checkerboard Lounge, Chicago 1981.}

CD/DVD et LP. Eagle Vision, enregistré le 22 novembre 1981 au Checkerboard Lounge de Chicago, sortie le 10 juillet 2012.

Elodie A. Grossi

$$
\begin{aligned}
& \text { "Well my mother told my father just before I was } \\
& \text { born } \\
& \text { 'I got a boy child comin', gonna be, gonna be a } \\
& \text { rollin' stone } \\
& \text { Sho' enough he's a rollin' stone..." } \\
& \text { Muddy Waters, couplet de "Rollin' Stone”, } 1950 .
\end{aligned}
$$

Le 22 novembre 1981, auréolés du succès de leurs récentes dates de tournée aux ÉtatsUnis, les Rolling Stones débarquèrent au Checkerboard Lounge, un club de blues du quartier de Bronzeville, enclave " africaine-américaine » du South Side de Chicago. Le groupe avait en effet décidé d'assister à ce qui fut l'une des dernières performance du légendaire Muddy Waters. Mick Jagger, Keith Richards et Ron Wood finirent par se livrer à l'exercice scénique, à la demande générale, partageant au coude à coude avec John Primer, Buddy Guy, Junior Wells et Lefty Dizz la minuscule scène du Checkerboard.

2 L'édition de la version restaurée de cette unique performance live, plus d'une trentaine d'années après son enregistrement, nous offre ainsi l'occasion de nous interroger sur le croisement des différents courants de musique populaire labellisés en tant que tel, ainsi que de réfléchir à ce qui est vu comme le « renouveau » du blues après l'explosion du rock des deux cotés de l'Atlantique, dont les Rolling Stones et les Beatles sont dans une certaine mesure les héritiers ${ }^{1}$. Il nous semble ainsi utile de ne pas appréhender ce disque comme un moment de rencontre entre deux traditions foncièrement distanciées dès leurs supposées " origines ", mais plutôt de souligner les continuités et influences réciproques de l'une à l'autre. 
Qu'est-ce qui forme ainsi le caractère exceptionnel de la rencontre entre les Rolling Stones, jouissant d'une immense notoriété et un des "patrons» du blues sur scène, Muddy Waters dans un obscur club de Chicago ? On peut bien sûr poser la question de l'organisation de la performance scénique conjointe et la spontanéité de l'arrivée des Rolling Stones dans le club. Était-ce un exercice prémédité et un acte de promotion sans précédant orchestré par les Rolling Stones afin de mesurer l'authenticité de leurs racines blues à la lumière de Muddy Waters, dans le club historiquement «africainaméricain» du South Side? Il peut être question de construire véritablement «l'authenticité » de la performance autour de la production d'une image racialisée du son blues, appréhendé historiquement comme un courant artistique «africainaméricain ", comme le rappelle David Grazian dans son ethnographie Blue Chicago ${ }^{2}$. Fait intéressant, John Szwed dans Crossovers: Essays on Race, Music and American Culture développe une théorie de "racialisation» du son Blues en citant l'exemple de Mick Jagger qui serait le symbole même de l'absorption de courants musicaux référencés comme "noirs" dans un courant de musique qu'il catégorise comme "blanche $»^{3}$. Appréhender les deux genres musicaux de cette manière contribue à consolider les deux courants musicaux en deux catégories fixes, perçues l'une par rapport à l'autre de manière antagoniste. De plus, cet argument comporte le risque de réitérer une construction réductrice du blues limité à une dimension qui se veut " raciale », tandis qu'elle intensifie sa production en tant que genre subordonné à la musique populaire rock dite «blanche ».

4 Il est intéressant d'appréhender la trajectoire historique du blues à Chicago à travers le paradigme de l'histoire sociale de la ville, et en partie celle de la Grande Migration, épisode qui désigne la migration du Sud au Nord au cours de la première moitié du $\mathrm{XX}^{\mathrm{e}}$ siècle de nombreuses familles "africaines-américaines ", dont celle de Muddy Waters, sous les conséquences implacables de la révolution industrielle en cours ${ }^{4}$. On peut également remarquer que la musique blues, parfois caractérisée par son prétendu fondement «sudiste" s'installe ainsi à Chicago, et évolue ainsi au rythme des influences multiples de la ville. Cette vision mouvante de la musique blues s'oppose en cela à la construction de l'authenticité performative qui place le blues dans un paradigme nostalgique et qui l'identifierait à un sempiternel passé romancé.

5 La dimension visuelle de la performance scénique est importante tout au long des dix chansons composant la setlist du concert réunissant Muddy Waters et Mick Jagger, Keith Richards et Ron Wood des Rolling Stones. Ce concert constitue un véritable oxymore visuel, dans l'atmosphère lourde et enfumée du club du South Side, soulignée par l'étroitesse de la scène. Perché sur le tabouret central, Muddy Waters enchaîne les titres, de "Got My Mojo Working " à "Baby Please Don't Go ", deuxième chanson du concert, sur laquelle il esquisse quelques accords sur sa Fender Telecaster, avant l'arrivée du groupe dans le club. Alors que l'arrivée du groupe est annoncée, on voit à l'écran fans et badauds se précipiter à l'entrée du Checkerboard. Le vigile tente, en vain, de les dissuader, tandis que les membres du groupe au complet passent sous les projecteurs et s'installent précipitamment à une table faisant face à la scène. La caméra balaie alors la scène du Checkerboard et effectue un gros plan sur le visage de Mick Jagger, qui se glisse sur une chaise en retrait, avant d'ôter son trench en tweed et de commander une tournée pour lui et ses complices. La scène attire l'attention de Muddy Waters, qui s'adresse à Mick Jagger d'un air amusé ("Take your time, no rushes »), avant de finalement l'interpeller d'un vindicatif «Mick Jagger up here!». Après 
quelques instants d'hésitation, le chanteur, mimant une moue amusée se glisse hors de son siège sous les applaudissements des clients alentours, passant de la semi-pénombre de la salle à la lumière feutrée de la scène. La voix tonitruante de Muddy Waters s'élève alors entre deux couplets, pour réclamer Keith Richards sur scène ("What about Keith?»), tandis que celui-ci enjambe une table, embrassant au passage la serveuse, cigarette à la lèvre. Muddy Waters se décide finalement à inviter Ron Wood sur la scène artisanale du Checkerboard, et ce dernier se précipite auprès des autres membres du groupe, verre à la main, alors que le club vibre à présent sous les accords tranchants de cinq Fender. Trois chansons plus tard, Muddy Waters semble avoir rajeuni de trente ans, délaissant son tabouret sur " Mannish Boy ", et chantant aux côtés de Mick Jagger, ravi d'accueillir Buddy Guy et Lefty Dizz parmi les musiciens sur scène.

6 En cela, le concert tient davantage de l'hommage que de la performance, hommage qui prend tout son sens lorsque l'on sait que le nom du groupe fut choisi par Brian Jones, guitariste et fondateur du groupe, en référence à la chanson « Rollin' Stones » reprise en 1950 par Muddy Waters. L'édition de ce live CD/DVD offre l'occasion de voir également émerger une influence rétrospective du rock par rapport au blues, moins habituelle que celle du blues au rock. Le blues, vu parfois seulement en relation au rock'n'roll prend ici toute son ampleur, aux cotés des Rolling Stones qui avaient à leurs débuts en partie amorcé sa diffusion auprès du public «blanc » britannique mainstream. Sur « Hoochie Coochie Man », alors que Mick Jagger chante «I was born for good luck », Muddy Waters lui donne la réplique en renchérissant d'une boutade par «I see that !» sur les accords des Stratocasters de Ron Wood et Keith Richards. Cet échange illustre d'une certaine manière la complexe relation d'ombre et lumière entre les Rolling Stones, groupe mainstream rock par excellence et Muddy Waters, véritable vétéran du blues qui tend à être connu d'un public plus confidentiel ${ }^{5}$.

7 Bien qu'elles furent restaurées en vue de la sortie du CD/DVD par Bob Clearmountain, ingénieur son de longue date des Rolling Stones, les bandes audio du concert conservent à la première écoute un son peu lisse, enregistrées dans les conditions d'un live sans équipement sonore optimal. Sans doute ce choix de restauration sonore cherche à mettre en avant le coté spontané du concert et contribue à construire ainsi une impression d'authenticité, corroborée par le montage d'images de qualité VHS qui proviennent d'archives vidéo. On ne peut alors s'empêcher de penser aux débuts des Rolling Stones sur scène en visionnant le DVD du live de 1981, en tout point similaire à la performance scénique de leurs débuts dans les clubs londoniens. Les Rolling Stones, comme d'autres groupes britanniques de leur génération sont le produit de la scène live, avant de devenir musiciens de studio, tout comme les Beatles qui occupèrent durant des années les petits clubs de Hambourg, où ils se firent la main sur quelques standards du blues et de rock n'roll américain ${ }^{6}$. Le concert au Checkerboard permet aux Rolling Stones d'effectuer une coupure après leurs éreintantes tournées des stades des années 1970 et 1980. Mick Jagger passe ainsi du statut du chanteur star à celui du fan stupéfait, ce qui contribue à donner une image alternative quelque peu en retrait des Rolling Stones à laquelle le public n'est guère habitué après les concerts pharaoniques de leurs tournées mondiales successives. Selon la rumeur ${ }^{7}$, le concert était tout arrangé et le manager du groupe aurait ainsi avancé 500 dollars au patron de l'époque du Checkerboard pour confirmer la venue des Rolling Stones une semaine avant le concert, dans l'intention de casser le côté prétentieux du son et des shows de l'époque et de donner une image plus intimiste du groupe sur scène. Réunir Muddy Waters et les 
Rolling Stones aurait été ainsi l'occasion de réconcilier le groupe avec son envahissante notoriété.

8 L'édition du disque Live at the Checkerboard Lounge, Chicago 1981 peut être lue comme une double entreprise : celle de resituer les Rolling Stones dans un courant de musique blues et de redessiner l'authenticité de leur son par rapport à celui de Muddy Waters, en veillant habilement à casser l'image de consommation mainstream du groupe de l'époque. Quoiqu'il en soit, les douze titres du disque renferment une incomparable vivacité et seront aussi bien appréciés par les fans inconditionnels du groupe, que par les férus du blues de Chicago.

Liste des morceaux :

DVD :

11 1) Sweet Little Angel

2) Flip Flop And Fly

3) Muddy Waters Introduction

4) You Don't Have To Go

5) Country Boy

6) Baby Please Don't Go

7) Hoochie Coochie Man

8) Long Distance Call

9) Mannish Boy

10) Got My Mojo Working

11) Next Time You See Me

12) One Eyed Woman

13) Baby Please Don't Go (Instrumental)

14) Blow Wind Blow

15) Champagne \& Reefer CD :

1) Introduction Instrumental

2) You Don't Have To Go

3) Baby Please Don't' Go

4) Hoochie Coochie Man

5) Long Distance Call

6) Mannish Boy

7) Got My Mojo Working

8) Next Time You See Me

9) One Eyed Woman

10) Blow Wind Blow

11) Champagne \& Reefer 


\section{NOTES}

1. Si les Rolling Stones sont souvent cités comme étant l'exemple le plus probant des groupes britanniques influencés par les standards de blues américain dès le tout début de leur carrière, on peut aisément identifier d'autres formations musicales de la même époque qui se font les héritiers de la tradition Blues Rock. Les Beatles par exemple se réclamaient au fil des interviews moins du blues que des pionniers du rock'n roll (Chuck Berry) et du folk (Bob Dylan), mais naviguèrent entre différents courants musicaux, dont les influences sont perceptibles dans un certain nombre de leurs titres. On peut ainsi citer la reprise des Beatles de « Matchbox » de 1964, standard de blues de 1927 de Blind Lemon Jefferson, ainsi qu'entre autres, les chansons "Yer Blues » et «Revolution » du duo John Lennon/Paul McCartney et «For You Blue » de George Harrison qui suivaient une progression évidente d'accords blues.

2. Voir David Grazian, Blue Chicago: The Search for Authenticity in Urban Blues Club, Chicago, The University of Chicago Press, 2005.

3. "The fact that, say, a Mick Jagger can today perform in the same tradition without blackface simply marks the detachment of culture from race and the almost full absorption of a black tradition into white culture ", dans John Szweed, Crossovers: Essays on Race, Music and American Culture, Philadelphia, University of Pennsylvania Press, 2006, 85.

4. Voir James Grossman, Land of Hope: Chicago, Black Southerners, and the Great Migration, Chicago, University of Chicago Press, 1991.

5. John Primer et Lefty Dizz ne sont d'ailleurs pas référencés sur l'album CD et le DVD, ce qui en dit long sur la reconnaissance et la commercialisation des artistes de musique considérée comme mainstream ou non.

6. Dans une autobiographie publiée en 2011, Keith Richards se remémore les débuts scéniques des Rolling Stones : "The spaces were small, which suited us. It suited Mick best of all. Mick's artistry was on display in these small venues, where there was barely space to swing a catperhaps more so than it ever was later... Give Mick Jagger a stage the size of a table and he could work it better than anybody, except maybe James Brown ", dans Life, 2010, Chapitre 4.

7. Pour les sources de cette rumeur, voir http://timeoutchicago.com/things-to-do/this-week-inchicago/15521761/muddy-waters-the-rolling-stones-live-at-the-checkerboard-

ou encore: http://www.suntimes.com/entertainment/music/16582852-421/when-the-rollingstones-muddy-waters-jammed-at-checkerboard.html

INDEX

Thèmes : La boîte à musique

\section{AUTEUR}

ELODIE A. GROSSI

Université Paris VII - Diderot, University of Chicago 